\title{
Detection Algorithm of Friction and Wear State of Large Mechanical and Electrical Equipment in Coal Mine based on C-SVC
}

\author{
Xinliang Wang ${ }^{\mathrm{a}, \mathrm{b}, *}$, Zhigang Guo ${ }^{\mathrm{c}}$, Jianlin Chen ${ }^{\mathrm{a}}$, Na Liu ${ }^{\mathrm{d}}$, and Wei Fang ${ }^{\mathrm{d}}$ \\ ${ }^{a}$ School of Physics and Electronic Information Engineering, Henan Polytechnic University, Jiaozuo, 454000, China \\ ${ }^{b}$ Hami Yuxin Energy Industry Research Institute Co., Ltd., Hami, 839000, China \\ ${ }^{c}$ School of Electrical Engineering and Automation, Henan Polytechnic University, Jiaozuo, 454000, China \\ ${ }^{d}$ Hami Vocational and Technical College, Hami, 839000, China
}

\begin{abstract}
The large-scale electromechanical equipment of coal mines has the characteristics of low speed, heavy loads, and complicated operation environment. Existing features, such as shape, color, and texture, are directly used to detect the friction and wear state of large mechanical and electrical equipment in coal mines, and the effect is not satisfactory. In this paper, a multivariate feature extraction algorithm based on maximum wear particles is proposed, and the C-SVC classifier model is constructed based on the extracted features. The simulation results show that compared with SVM (Support Vector Machine) and the decision tree algorithm, the model of C-SVC classifier based on the multiplex feature of the largest block wear particles has better classification accuracy, better generalization ability, and better robustness.
\end{abstract}

Keywords: ferrography images analysis; feature extraction; maximum wear particle; C-SVC classifier

(Submitted on October 21, 2018; Revised on November 24, 2018; Accepted on December 22, 2018)

(C) 2019 Totem Publisher, Inc. All rights reserved.

\section{Introduction}

At present, oil ferrography analysis technology has been widely used in the fault detection of large-scale mechanical equipment. Image processing technology can be used to analyze ferrography images more accurately, and then the feature information can be extracted [1]. With the feature matrix, the ferrography images are classified by pattern recognition technology. In 2005, Kang et al. [2] proposed an improved neural network algorithm by modifying the weights of the hidden layer and output layer separately. The wear particle classifier was designed to identify the wear particle type by establishing the wear particle analysis model. In 2009, Qiu et al. [3] put forward the application of oil analysis technology to the daily maintenance of modern papermaking machinery to test the failure of the equipment. In 2010, Zhang et al. [4] put forward a neural network expert system for wear particle recognition based on the principle of neural network expert system, which improved the ability of feature recognition of wear particles and the efficiency of ferrography analysis. In 2016 , He et al. [5] used oil analysis technology to diagnose gear failure of the reducer of the extruder and effectively detected the wear and pitting phenomenon caused by poor lubrication in the extruder.

The literature described above has been able to detect the fault state of large-scale mechanical equipment effectively. However, there are few studies on the fault state detection of large mechanical equipment based on automatic ferrography image analysis. The investment of large coal mine mechanical and electrical equipment is huge. For both fixed and mining equipment, most of them are low-speed and heavy-duty equipment. The equipment runs in a bad environment and the load changes greatly, which will cause serious wear to the equipment. If the lubrication condition of the equipment is poor, the equipment will be damaged or even scrapped, seriously affecting the safety of coal mine production. Therefore, realizing automatic fault state detection of large mechanical and electrical equipment based on automatic analysis of ferrography images is the main problem to be solved in this paper.

The structure of coal mine machinery and equipment is complex, and the maintenance cost is high. Extensive research

* Corresponding author.

E-mail address: junci158@163.com 
data show that over $80 \%$ of equipment failures are caused by wear [6]. Due to the complex working environment, equipment failure will seriously affect the normal working schedule, and the loss will be inestimable. The mature application of oil ferrography analysis technology is of great significance to prevent accidents in advance [7]. In 2014, Fu [8] demonstrated the application of the oil analysis method in the lubrication and maintenance of coal mine air compressors and successfully verified the application of oil analysis technology in the monitoring and fault diagnosis of coal mine air compressors. In 2015, the management mechanism of coal mine equipment based on modern oil analysis technology was put forward in literature [9]. Through oil sample analysis, the quality of oil was qualitatively analyzed, and the condition monitoring of key equipment was realized. In the same year, Huang [10] introduced oil monitoring and analysis technology into electromechanical maintenance. By analyzing the oil, the failure rate of the equipment was reduced and the service life of the equipment was prolonged. In 2016, Xie et al. [11] carried out ferrography analysis of lubricating oil for large coal mine equipment. Through qualitative and quantitative analysis of spectrum sheets, the operating state of the equipment can be obtained and the equipment fault can be diagnosed early.

In the literature above, the fault state detection of large mechanical and electrical equipment in coal mines is mainly based on artificial subjective analysis of ferrography images. In this paper, the support vector machine (SVM) classification model based on C-SVC [12] is constructed by introducing the multiplex feature of the largest abrasive particle, and the ferrographic analysis image of large and medium-sized coal mining equipment is automatically analyzed to complete the fault state detection, which has good practical value.

\section{Multiple Feature Extraction Algorithm based on Maximum Block Wear Particles}

\subsection{Data Acquisition Module}

In this paper, the ferrography images samples are obtained from Henan Pingdingshan Pingmei Shenma Group Equipment Leasing Branch. The company is mainly engaged in the analysis of friction and wear of large mechanical and electrical equipment in coal mines, oil state analysis, etc., with rich experience and high reliability of the iron spectrum samples provided. It provides an abundant database for the automatic detection of friction and wear state of large mechanical and electrical equipment in coal mines.

\subsection{Image Preprocessing Module}

Ferrogram images should undergo grayscale processing first, and the grayscale image will be segmented again, which will be more favorable for segmentation. The feature information of the wear particles can be better extracted from the ferrography images by image preprocessing. The effect of segmentation is shown in Figure 1.

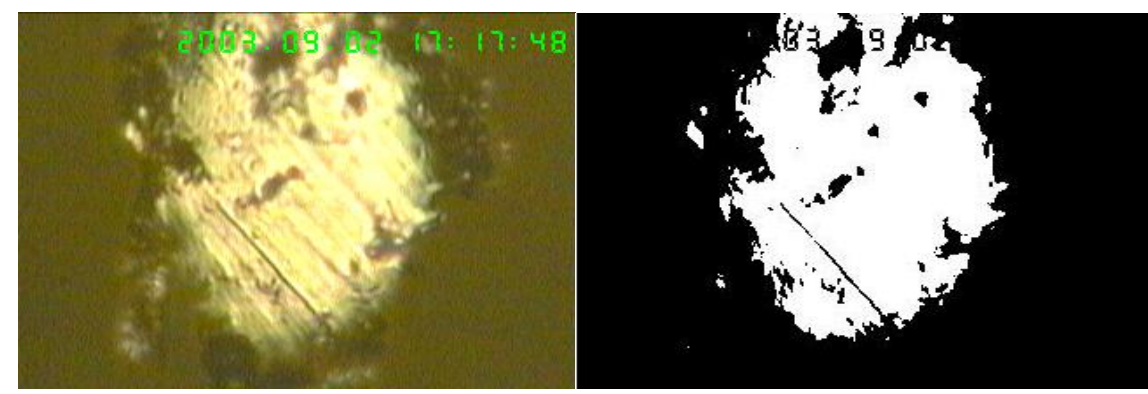

Figure 1. Comparison between original image and segmented image

\subsection{Multiple Feature Selection and Extraction Algorithm for Maximum Wear Particles}

The shape, color, texture, and other features of wear particles in ferrography images were selected and extracted in literature [13-14], but the existing features such as shape, color, and texture were directly used to detect the friction and wear status of large mechanical and electrical equipment in coal mines. The effect was unsatisfactory. Due to the complex working environment and serious dust pollution of large-scale mechanical and electrical equipment in coal mines, the direct use of existing characteristics to detect the wear and friction state of large electromechanical equipment in coal mines is not ideal.

In this paper, according to the operation characteristics of large mechanical and electrical equipment in coal mines, the largest wear particles in abnormal wear images are large in area and complex in surface texture features, and the largest wear particles in normal images are usually smaller than those in abnormal images. Based on the features of shape, color, 
texture, and so on, the features of area, perimeter, density, and deformity of the largest wear particles are proposed to construct the corresponding classifier model. The friction and wear state detection of coal mine electromechanical equipment is completed, and the accuracy of friction and wear state detection is improved effectively on the basis of existing features.

The specific algorithms for the multiplex characteristics of the wear particles are as follows:

Step 1 Identify all independent wear particles in the image based on the eight-connected rule, and all the wear particles are arranged in sequence number from 1. Assuming the number of independent wear particles is $N$, the initial values of $m_{j}$ (Maximum wear particle area) and $l_{z}$ (Maximum wear particle perimeter) are set to 0 , and the initial values of $i$ are set to 1 .

Step 2 Calculate the corresponding area and perimeter for the $i$ of the wear particle, and perform step 3.

Step 3 If $m_{j}<S$, then $m_{j}=S$ and $l_{z}=Z$; otherwise, the values of $m_{j}$ and $l_{z}$ are not updated.

Step 4 If $i<N$ and $i=i+1$, perform step 2; otherwise, the area and circumference of the maximum wear particles are saved in the current $m_{j}$ and $l_{z}$, and perform step 5 .

Step 5 Calculate the density $C$ based on $m_{j}$ and $l_{z}$, and $C=l_{z}^{2} / m_{j}$. The densification of the largest wear particles is also called complexity, which represents the discrete state in the unit region. When the value of $C$ is larger, it shows that the circumference of the unit area is larger, that is, the region of the largest wear particles is discrete and the shape is more complex. On the contrary, when the $C$ value is small, the shape of the largest wear particle is simple.

Step 6 Calculate the deformity degree $J$ based on $m_{j}$ and $l_{z}$, and $J=l_{z} / m_{j}^{2}$. The deformities of the largest wear particles reflect the morphological characteristics of the abrasive particles and are determined by the relationship between the area and the circumference of the abrasive particles. When the J value is small, the edges of the particles are smooth and round in shape. On the contrary, the grain boundary is irregular or the grain shape is slender.

\section{Design of C-SVC Classifier Model based on Multi Feature of Maximum Block Wear Particles}

\subsection{C-SVC Model Principle}

C-SVC [12] is a classification model of SVM. The introduction of the C-SVC model can classify samples more accurately to achieve the purpose of experiment. Assuming that the normal vector of the hyperplane is $w$, in C-SVC the hyperplane under ideal conditions minimizes $\frac{1}{2}\|w\|^{2}$. In the C-SVC model, two new variables $\left(\varepsilon_{i}\right.$ and $C$ ) should be introduced. $\varepsilon_{i}$ is a relaxation variable that is introduced to solve the problem when a certain point is far away from a group. For this kind of outlier, we introduce the relaxation variable as a parameter in order to better optimize the function model. The influence of noise point is reduced to the minimum. $C$ is the penalty factor. Its size depends on the importance of outliers, and the greater the $C$ value, the more important the outliers and the greater the impact on the classification results. At this point, the problem that requires the solution is under the constraint $y_{i}\left(w \cdot x_{i}+b\right) \geq 1-\varepsilon_{i},(i=1,2, \cdots, n), \varepsilon_{i} \geq 0$. Calculate $\min _{w}\left(\frac{1}{2}\|w\|^{2}\right)+\mathrm{C} \sum_{i=1}^{n} \varepsilon_{i}$.

\subsection{Classifier Feature}

In this paper, based on the diversity of wear particles (the area of the largest wear particles, circumference of the largest wear particles, characteristics of density, and deformity of the largest block wear particles), the existing ferrography images wear particle area [13], ferrography images Euler number [13], ferrography images voids, color mean value of ferrography image [14], color variance of ferrography images [14], texture energy matrix of ferrography images [13], texture moment of inertia of ferrography images [13], texture correlation matrix of ferrography images [13], and C-SVC classifier model are constructed. The classification process is as follows:

The data extracted based on the above features is used as the training sample of the C-SVC model. The training sample 
is $\left\{\left(x_{i}, y_{i}\right)\right\}_{i=1,2, \cdots, n}$, where $x_{i} \in R^{22}, x_{i}$ represents sample characteristics, and $R^{22}$ is a 22-dimensional feature space. $x_{i}$ for each of the feature space contains 22 pluralistic features. $x_{i 1}$ represents the area of wear particles in ferrography images; $x_{i 2}$ represents the Euler number of ferrography images; $x_{i 3}$ represents the number of wear particles in ferrography images; $x_{i 4}$ represents the voidage rate of ferrography images; $x_{i 5}, x_{i 6}, x_{i 7}$ respectively represent the RGB mean of ferrography images; $x_{i 8}, x_{i 9}, x_{i 10}$ respectively represent the RGB variance of ferrography images; and $x_{i 11}, x_{i 12}, x_{i 13}, x_{i 14}$ respectively represent the mean value of texture energy, entropy, moment of inertia, and correlation matrix of ferrography images. $x_{i 15}, x_{i 16}, x_{i 17}, x_{i 18}$ respectively represent the standard deviation in the texture energy, entropy, moment of inertia, and correlation matrix of ferrography images. $x_{i 19}, x_{i 20}$ respectively represent the area and circumference of the largest worn particle in the ferrography images. $x_{i 21}$ represents the density of the largest worn particles in ferrography images. $x_{i 22}$ represents the deformities of the largest worn particles in ferrography images. $y_{i} \in\{-1,1\}$ represents sample labels, -1 represents no abnormal sample grouping, 1 represents abnormal wear sample grouping, and the number of samples is $n$.

\subsection{Kernel Function Selection}

The feature data extracted in this paper have a total of 22 dimensions, which obviously show linear inseparability. In order to solve this problem, the kernel function is introduced to calculate linear inseparability. The kernel function maps the linear inseparable data in the low-dimensional feature space to a high-dimensional feature space, which makes the data separable in the feature space. In this paper, the Gaussian (RBF) kernel function is chosen. $K\left(x_{i}, x_{j}\right)=\exp \left(-\frac{\left\|x_{i}-x_{j}\right\|^{2}}{\sigma^{2}}\right)$, where $\sigma$ is the width parameter of the function and controls the radial action range of the function. To facilitate the calculation of parameters, set $g=1 / \sigma^{2}$, and then the kernel function becomes $K\left(x_{i}, x_{j}\right)=\exp \left(-g\left\|x_{i}-x_{j}\right\|^{2}\right)$.

\subsection{Classification Training and Testing Process based on C-SVC}

The sample data training set eliminates the constraint condition by introducing the Lagrange multiplier $\alpha_{i}, \gamma_{i}$. As indicated in Equation (1),

$$
\begin{aligned}
\Lambda\left(w, b, \alpha_{1}, \alpha_{2}, \cdots, \alpha_{n}, \gamma_{1}, \gamma_{2}, \cdots, \gamma_{n}\right) & =\frac{1}{2}\|w\|^{2}+C \sum_{i=1}^{n} \varepsilon_{i}-\sum_{i=1}^{n} \alpha_{i} \cdot\left(y_{i} \cdot \sum_{k=1}^{22}\left(w_{k} \cdot x_{i k}+b\right)-1+\varepsilon_{i}\right)-\sum_{i=1}^{n} \gamma_{i} \varepsilon_{i} \\
& =\frac{1}{2} w^{2}+C \sum_{i=1}^{n} \varepsilon_{i}-\sum_{i=1}^{n} \alpha_{i} y_{i} \sum_{k=1}^{22} w_{k} x_{i k}-b \sum_{i=1}^{n} \alpha_{i} y_{i}+\sum_{i=1}^{n} \alpha_{i}-\sum_{i=1}^{n} \alpha_{i} \varepsilon_{i}-\sum_{i=1}^{n} \gamma_{i} \varepsilon_{i}
\end{aligned}
$$

Equation (1) is derived from the parameters $w, b$, and $\varepsilon_{i}$, and the derivative is 0.

$$
\begin{gathered}
\frac{\partial \Lambda\left(w, b, \alpha_{1}, \alpha_{2}, \cdots, \alpha_{n}, \gamma_{1}, \gamma_{2}, \cdots, \gamma_{n}\right)}{\partial w}=w-\sum_{i=1}^{n} \alpha_{i} y_{i} x_{i}=0 \\
\frac{\partial \Lambda\left(w, b, \alpha_{1}, \alpha_{2}, \cdots, \alpha_{n}, \gamma_{1}, \gamma_{2}, \cdots, \gamma_{n}\right)}{\partial b}=-\sum_{i=1}^{n} \alpha_{i} y_{i}=0
\end{gathered}
$$

and

$$
\frac{\partial \Lambda\left(w, b, \alpha_{1}, \alpha_{2}, \cdots, \alpha_{n}, \gamma_{1}, \gamma_{2}, \cdots, \gamma_{n}\right)}{\partial \varepsilon_{i}}=C-\alpha_{i}-\gamma_{i}=0
$$

Therefore, 


$$
w=\sum_{i=1}^{n} \alpha_{i} y_{i} x_{i}, \sum_{i=1}^{n} \alpha_{i} y_{i}=0, \text { and } C-\alpha_{i}-\gamma_{i}=0
$$

Then,

$$
\begin{aligned}
& \Lambda\left(w, b, \alpha_{1}, \alpha_{2}, \cdots, \alpha_{n}, \gamma_{1}, \gamma_{2}, \cdots, \gamma_{n}\right) \\
& =\frac{1}{2} w^{2}+C \sum_{i=1}^{n} \varepsilon_{i}-\sum_{i=1}^{n} \alpha_{i} y_{i} \sum_{k=1}^{22} w_{k} x_{i k}-b \sum_{i=1}^{n} \alpha_{i} y_{i}+\sum_{i=1}^{n} \alpha_{i}-\sum_{i=1}^{n} \alpha_{i} \varepsilon_{i}-\sum_{i=1}^{n} \gamma_{i} \varepsilon_{i} \\
& =\frac{1}{2} \sum_{i=1}^{n} \alpha_{i} y_{i} x_{i} \sum_{j=1}^{n} \alpha_{j} y_{j} x_{j}-\sum_{i=1}^{n} \alpha_{i} y_{i} x_{i} \sum_{j=1}^{n} \alpha_{j} y_{j} x_{j}-b \sum_{i=1}^{n} \alpha_{i} y_{i}+\sum_{i=1}^{n} \alpha_{i} \\
& =-\frac{1}{2} \sum_{i=1}^{n} \alpha_{i} y_{i} x_{i} \sum_{j=1}^{n} \alpha_{j} y_{j} x_{j}+\sum_{i=1}^{n} \alpha_{i}=-\frac{1}{2} \sum_{i=1, j=1}^{n} \alpha_{i} \alpha_{j} y_{i} y_{j} \sum_{k=1}^{22}\left(x_{i k} \cdot x_{j k}\right)+\sum_{i=1}^{n} \alpha_{i}
\end{aligned}
$$

Bring the RBF kernel function $K\left(x_{i}, x_{j}\right)=\exp \left(-\frac{\left\|x_{i}-x_{j}\right\|^{2}}{\sigma^{2}}\right)$ into Equation (2) to obtain Equation (3).

$$
\begin{aligned}
\Lambda\left(w, b, \alpha_{1}, \alpha_{2}, \cdots, \alpha_{n}, \gamma_{1}, \gamma_{2}, \cdots, \gamma_{n}\right) & =\frac{1}{2} w^{2}+\mathrm{C} \sum_{i=1}^{n} \varepsilon_{i}-\sum_{i=1}^{n} \alpha_{i} y_{i} \sum_{k=1}^{22} w_{k} x_{i k}-b \sum_{i=1}^{n} \alpha_{i} y_{i}+\sum_{i=1}^{n} \alpha_{i}-\sum_{i=1}^{n} \alpha_{i} \varepsilon_{i}-\sum_{i=1}^{n} \gamma_{i} \varepsilon_{i} \\
& =\frac{1}{2} \sum_{i=1}^{n} \alpha_{i} y_{i} x_{i} \sum_{j=1}^{n} \alpha_{j} y_{j} x_{j}-\sum_{i=1}^{n} \alpha_{i} y_{i} x_{i} \sum_{j=1}^{n} \alpha_{j} y_{j} x_{j}-b \sum_{i=1}^{n} \alpha_{i} y_{i}+\sum_{i=1}^{n} \alpha_{i} \\
& =-\frac{1}{2} \sum_{i=1}^{n} \alpha_{i} y_{i} x_{i} \sum_{j=1}^{n} \alpha_{j} y_{j} x_{j}+\sum_{i=1}^{n} \alpha_{i}=-\frac{1}{2} \sum_{i=1, j=1}^{n} \alpha_{i} \alpha_{j} y_{i} y_{j} K\left(x_{i}, x_{j}\right)+\sum_{i=1}^{n} \alpha_{i}
\end{aligned}
$$

Eventually, $\min \left(\frac{1}{2}\|w\|^{2}\right)+C \sum_{i=1}^{n} \varepsilon_{i}$ is converted to

$$
\begin{aligned}
& \max _{\alpha}-\frac{1}{2} \sum_{i=1, j=1}^{n} \alpha_{i} \alpha_{j} y_{i} y_{j} k\left(x_{i}^{22} \cdot x_{j}^{22}\right)+\sum_{i=1}^{n} \alpha_{i} \\
& \text { s.t. } \quad 0 \leq \alpha_{i} \leq C, i=1,2, \cdots, n, \quad \sum_{i=1}^{n} \alpha_{i} y_{i}=0
\end{aligned}
$$

Based on Equation (4), the C-SVC classification model based on the diversity of the maximum wear particles can be obtained. Assume that the sample data for the test set is $\left\{\left(x_{t}, y_{i}\right)\right\}_{t=1,2, \ldots, m}, x_{t} \in R^{22}$, and $R^{22}$ is a 22-dimensional feature space. $y_{i} \in\{-1,1\}$ represents sample labels, -1 represents no abnormal sample grouping, 1 represents abnormal wear sample grouping, and the number of samples is n. Bring $x_{t}$ and $w=\sum_{i=1}^{n} a_{i} y_{i} x_{i}^{22}$ into the model.

$$
\begin{aligned}
& y_{t}=w x_{t}^{22}+b=\left(\sum_{i=1}^{n} \alpha_{i} y_{i} x_{i}^{22}\right) x_{t}^{22}+b=\sum_{i=1}^{n} \alpha_{i} y_{i} k\left(x_{i}^{22} \cdot x_{t}^{22}\right)+b \\
& \text { s.t. } 0 \leq \alpha_{i} \leq C, i=1,2, \cdots, n
\end{aligned}
$$

According to the calculation results of Equation (5), the category of test samples can be obtained, and the trained classifier model can be used to classify the test samples.

\subsection{Parameter Optimization Process}

In order to further improve the generalization ability and prediction ability of the C-SVC model, the cross-validation method is used to optimize the parameters of the C-SVC model. The parameters $(c, g)$ of the most appropriate pre-training set are 
found by training based on the multiplex features of the largest block wear particles, the best model is obtained, and the test set is classified and verified by the model. Cross verification is an important method to verify the good performance of the classifier. K-fold cross validation [15] is a kind of cross verification. Because of its strong computing ability and short time, it is widely used. In this paper, five-fold cross-validation is used to optimize the training samples of the C-SVC model, and the $(c, g)$ parameter with the highest accuracy of the C-SVC model is used as the optimal parameter to classify the test set.

\section{Simulation Analysis}

\subsection{Experimental Environment and Data}

This experiment is based on MATLAB 2013 a platform, using a simple and practical toolbox libsvm developed by Professor Lin Zhiren of Taiwan University. The experimental data are obtained from Pingdingshan Pingmei Shenma Group Equipment Leasing Branch. All the experimental images are classified and summarized according to the working experience of the company in the past ten years. After all the features are extracted, a 23-dimensional ferrography images information matrix is formed, which includes one dimensional label data $y=-1, y=1$ and 22-dimensional feature data.

In order to facilitate the subsequent experiments, the 23 dimensional ferrography images information matrix should be normalized. The normalized range is $(-1,1)$. When the normalized data is classified, the time required will be greatly reduced, and the efficiency of the C-SVC model will be improved. After normalization, we need to divide the test set and the training set. The images used in this experiment are non-abnormal and abnormal wear. There are 50 ferrography images without abnormal wear and 56 ferrography images with abnormal wear. A total of ten groups of sample data are extracted randomly from each of the two classes, and a test set with 20 groups of data is formed. The remaining data are automatically classified into training sets. Then, the C-SVC model is trained by the training set, and the optimal parameters are obtained. Establish the best classification model. The process is repeated ten times. Finally, the average accuracy of ten experiments is used as the classification result of C-SVC classifier model based on the maximum wear particles.

\subsection{Model Performance Analysis}

In this paper, the parameters are optimized by five-fold cross-validation. When the parameters $c$ and $g$ are optimized, the range of the two parameters is set in the range of -10 to 10 with step 0.2 . The results of parameter optimization and the corresponding prediction accuracy of C-SVC model are shown in Figure 2. From Figure 2, we can see that the classification accuracy will change as $(c, g)$ changes. In Figure 2, the optimal parameters and the best classification accuracy for the current training set are presented.
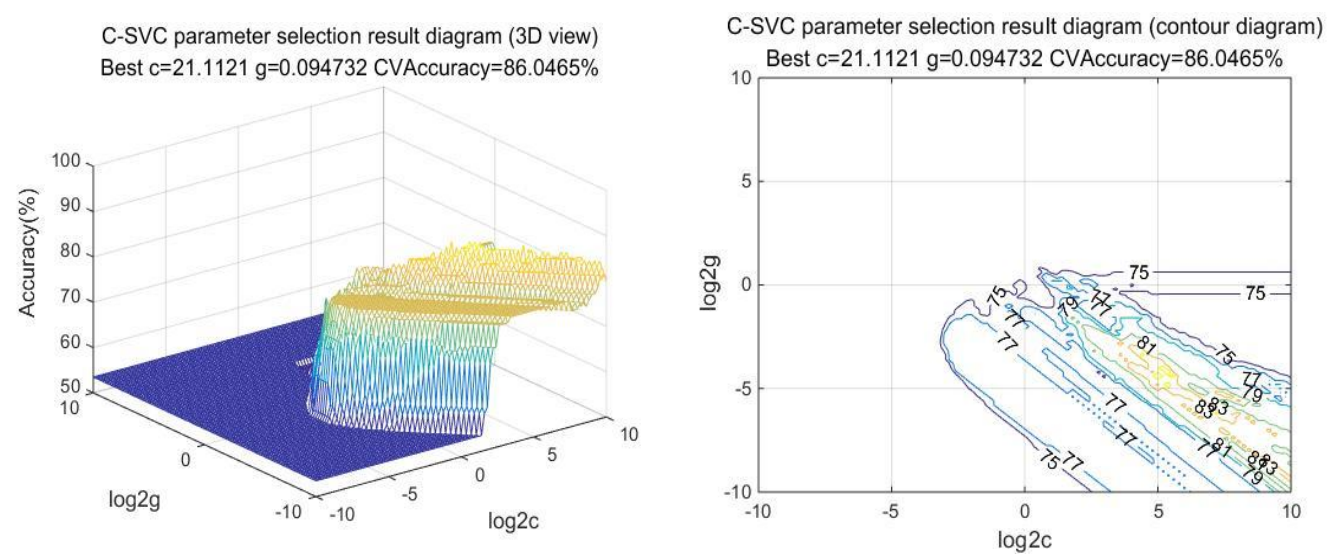

Figure 2. C-SVC parameter selection result diagram (3D view) and (contour diagram)

The results of C-SVC model classification based on the characteristics of maximum block wear particles are shown in Table 1. Table 1 shows that the classifier based on decision tree is time-consuming and the result is poor. The SVM model based on the multiplex characteristics of the largest wear particles has a poor classification effect because it does not consider the optimization of parameters. The C-SVC model, which does not contain the characteristics of maximum wear particles, is constructed. Although the parameter optimization is considered, the classification effect is not ideal because it does not introduce the multiplex feature based on the largest block wear particles. In this paper, the C-SVC model based on the multiple features of the largest wear particles is introduced, and the parameters are optimized to improve the classification accuracy and achieve a better classification effect. It has good practical value. 
Table 1. Comparison of classifier model classification results

\begin{tabular}{|c|c|c|c|}
\hline Classifier type & Normal & Inordinate wear & Total \\
\hline Decision tree & $60 \%$ & $60 \%$ & $60 \%$ \\
\hline $\begin{array}{l}\text { SVM model based on the multiplex characteristics of the maximal } \\
\text { wear particles }\end{array}$ & $50 \%$ & $80 \%$ & $65 \%$ \\
\hline $\begin{array}{l}\text { C-SVC model without multiplex characteristics of maximum wear } \\
\text { particles }\end{array}$ & $70 \%$ & $80 \%$ & $75 \%$ \\
\hline $\begin{array}{l}\text { C-SVC model based on the multiplex characteristics of the maximal } \\
\text { wear particles }(c=21.11, g=0.09)\end{array}$ & $90 \%$ & $100 \%$ & $95 \%$ \\
\hline
\end{tabular}

\subsection{Model Robustness Analysis}

There are 106 groups of sample data in this paper, and ten groups of data are randomly selected from two classes to form a test set of 20 groups of data. The remaining data are automatically classified into training sets. Then, the C-SVC model is trained by the training set, the optimal parameters are obtained, and the optimal classification model is established. Eight CSVC classifier models can be obtained by repeating the process eight times. The optimal parameters of the eight classifier models are shown in Table 2, and the classification accuracy of each model is shown in Table 3.

Table 2. Optimal parameters of eight models

\begin{tabular}{|c|c|c|}
\hline Number of experiments & Best $C$ & Best $g$ \\
\hline 1 & 24.2515 & 0.0474 \\
\hline 2 & 27.8576 & 0.0544 \\
\hline 3 & 42.2243 & 0.0272 \\
\hline 4 & 16 & 0.0718 \\
\hline 5 & 2.2974 & 0.5743 \\
\hline 6 & 21.1121 & 0.0473 \\
\hline 7 & 48.5029 & 0.0473 \\
\hline 8 & 14.25 & 0.05 \\
\hline
\end{tabular}

Table 3. Model classification accuracy

\begin{tabular}{|c|c|c|}
\hline Number of experiments & Normal & Inordinate wear \\
\hline 1 & $90 \%$ & $100 \%$ \\
\hline 2 & $80 \%$ & $100 \%$ \\
\hline 3 & $80 \%$ & $100 \%$ \\
\hline 4 & $80 \%$ & $80 \%$ \\
\hline 5 & $80 \%$ & $80 \%$ \\
\hline 6 & $80 \%$ & $100 \%$ \\
\hline 7 & $80 \%$ & $90 \%$ \\
\hline 8 & $90 \%$ & $90 \%$ \\
\hline Total & $82.5 \%$ & $92.5 \%$ \\
\hline
\end{tabular}

Table 3 shows that the average accuracy of eight C-SVC classification models is $82.5 \%$ and the average accuracy of abnormal wear is $92.5 \%$. The classification effect is good. It can be seen that the C-SVC model based on the multiplex characteristics of the largest block wear particles can effectively complete the classification of abnormal wear, and the model has good stability and robustness.

In order to verify the model's generalization ability and robustness better, a group of classification models are randomly selected from the above eight classification models, and a new classification experiment is carried out with the trained classification model without changing the parameters of the model. All sample data are reallocated randomly to form five new test sets. The first four test sets contain ten groups of abnormal data and ten groups of abnormal wear data. The fifth set contains ten groups of abnormal data and 16 groups of abnormal wear data. The test sets are classified by randomly selected classification models, and the classification results are shown in Table 4.

Table 4. Model classification accuracy

\begin{tabular}{|c|c|c|}
\hline Number of experiments & Normal & Inordinate wear \\
\hline 1 & $80 \%$ & $100 \%$ \\
\hline 2 & $90 \%$ & $100 \%$ \\
\hline 3 & $90 \%$ & $80 \%$ \\
\hline 4 & $90 \%$ & $90 \%$ \\
\hline 5 & $90 \%$ & $100 \%$ \\
\hline Total & $88 \%$ & $94 \%$ \\
\hline
\end{tabular}


Table 4 shows that the average accuracy of five C-SVC classification models without abnormal classification is 88 and the abnormal wear classification is 94 . The classification effect is good. It can be seen that the C-SVC model based on the multiplex characteristics of the largest block wear particles has better stability, strong generalization ability, and better robustness.

\section{Conclusions}

In this paper, a C-SVC classification optimization algorithm based on the multiplex features of the largest block wear particles is proposed, and the two types of ferrography images are classified by using the C-SVC classification model constructed by the algorithm. This method extracts the features of ferrography images in detail and studies the features of the largest particle to improve the diversity of features. The experimental results show that this method has strong generalization ability, robustness, high classification accuracy, and practical value. By comparing with the decision tree and the model without parameter optimization, it is found that the algorithm model has great advantages, not only with high classification accuracy, but also with less time required for classification. Compared with the C-SVC model, which does not contain the multiplex characteristics of the maximum wear particles, it is found that the characteristics of the maximum wear particles have a relatively large influence on the classification process. The algorithm model can provide strong support for the work of examiners, help examiners complete the identification and classification of fault images, and improve the ability of examiners to analyze and distinguish ferrography images.

\section{Acknowledgements}

This paper is supported by the Support Project of Science and Technology for Xinjiang Autonomous Region (No. 2018E02073), Doctor Fund of Henan Polytechnic University (No. B2012-073), Key Lab of Mine Informatization, Henan Polytechnic University (No. KY2015-08), Key Science and Technology Program of Henan Province (No. 172102210274), 2018 Annual Funding Plan for Key Scientific Research Projects in Henan Universities (No. 18A470013), and Fundamental Research Funds for the Universities of Henan Province (No. NSFRF170925).

\section{References}

1. H. L. Li, "Research on Ferrographic Image Segmentation and Wear Particle Feature Extraction Technology," Nanjing University of Aeronautics and Astronautics, Nan Jing, 2009

2. J. L. Kang, Y. P. Lu, and Y. S. Zhou, "Wear Particle Recognition with Improved BP Algorithm," LUBRICATION ENGINEERING, Vol. 3, pp. 41-42, 2004

3. R. H. Qiu, H. Zhang, and X. R. Zhang, "Oil Analysis Technique and its Application in Modern Paper Making Machinery Fault Diagnosis," Transactions of China Pulp and Paper, Vol. 24, No. 3, pp. 121-126, 2009

4. D. Zhang and P. J. Liang, "Design of the Expert System for Wear Particle Recognition based on Neural Network," Equipment Manufacturing Technology, Vol. 11, pp. 38-40, 2010

5. Z. R. He, Z. W. Sun, Z. N. Xuan, and Z. H. Duan, "Fault Diagnosis of the Gearbox of Petrochemical Extrusion Granulation Unit based on Oil Analysis Technology," Petro-Chemical Equipment Technology, Vol. 37, No. 1, pp. 15-18, 2016

6. E. Zhang, "Ferrography Technology and its Industrial Application,” Xi'an Jiao Tong University Press, Xi'an, 2001

7. Q. Li, T. G. Liu, C. Zhang, J. B. Zhao, and H. C. Zhang, "Study on Wear Condition Monitoring of Coal Mine Machinery by Comprehensive Ferrography Analysis Method," Coal Technology, No. 36, pp. 291-293, 2017

8. Z. X. Fu, "Compressor Oil Analysis Techniques in Coal Lubrication and Maintenance Management in Use," Coal Mine Machinery, Vol. 35, No. 4, pp. 183-184, 2014

9. X. J. Qin, “Application of Modern Oil Analysis Technology in Coal Mine Equipment Management," Science and Technology Innovation and Application, No. 18, pp. 153-153, 2015

10. J. R. Huang, "Application of Oil Analysis Technology in Monitoring of Coal Mine Operating Equipment," Electromechanical Information, No. 18, pp. 84-85, 2015

11. X. M. Xie and G. H. Liang, "Application of Iron Spectrum Analysis in Large Equipment of Coal Mine," ENERGY AND ENERGY CONSERVATION, No. 10, pp. 184-185, 2016

12. V. Vapnik, "The Nature of Statistical Learning Theory," Springer, New York, pp. 123-167, 2000

13. F. Wang, "Study on the Ferrography Wear Particle With Image Processing Technology," Wuhan University of Technology, Wuhan, 2005

14. L. J. Qiu, "Based on Support Vector Machine Ferrography Image Recognition Technology," Taiyuan University of Technology, 2015

15. M. M. Dundar, "A Cost-Effective Semisupervised Classifier Approach with Kernels," IEEE Transactions on Geoscience and Remote Sensing, Vol. 42, No. 1, pp. 264-270, 2004

Xinliang Wang is an associate professor in the School of Physics and Electronic Information Engineering at Henan Polytechnic University. His current research interests include smart power grids, cloud computing, and big data. 
Zhigang Guo is a graduate student in the School of Electrical Engineering and Automation at Henan Polytechnic University. His research interests include smart power grids, cloud computing, and big data.

Weiguo Li works in the Pingmei Shenma Group Equipment Leasing Branch. His research interests include cloud computing and big data.

Jianlin Chen is a student in the School of Physics and Electronic Information Engineering at Henan Polytechnic University.

Na Liu works at Hami Vocational and Technical College. Her research interests include cloud computing and big data.

Wei Fang works at Hami Vocational and Technical College. His research interests include smart power grids, cloud computing, and big data.

Xuebin Liu works at Hami Vocational and Technical College. His research interests include smart power grids, cloud computing, and big data.

Jun Wu works in the School of Physics and Electronic Information Engineering at Henan Polytechnic University. His current research interests include smart power grids, cloud computing, and big data. 